\title{
PDLIM2 restricts Th1 and Th17 differentiation and prevents autoimmune disease
}

\author{
Zhaoxia Qu ${ }^{1,2}$, Jing Fu ${ }^{1,2}$, Huihui Ma ${ }^{1,3}$, Jingjiao Zhou ${ }^{1,2}$, Meihua Jin ${ }^{1,2}$, Markus Y Mapara ${ }^{1,3}$, Michael J Grusby ${ }^{4}$ \\ and Gutian Xiao ${ }^{1,2^{*}}$
}

\begin{abstract}
Background: PDLIM2 is essential for the termination of the inflammatory transcription factors NF-KB and STAT but is dispensable for the development of immune cells and immune tissues/organs. Currently, it remains unknown whether and how PDLIM2 is involved in physiologic and pathogenic processes.

Results: Here we report that naive PDLIM2 deficient $C D 4^{+} T$ cells were prone to differentiate into Th1 and Th17 cells. PDLIM2 deficiency, however, had no obvious effect on lineage commitment towards Th2 or Treg cells. Notably, PDLIM2 deficient mice exhibited increased susceptibility to experimental autoimmune encephalitis (EAE), a Th1 and/or Th17 cell-mediated inflammatory disease model of multiple sclerosis (MS). Mechanistic studies further indicate that PDLIM2 was required for restricting expression of Th1 and Th17 cytokines, which was in accordance with the role of PDLIM2 in the termination of NF-KB and STAT activation.
\end{abstract}

Conclusion: These findings suggest that PDLIM2 is a key modulator of T-cell-mediated immune responses that may be targeted for the therapy of human autoimmune diseases.

\section{Background}

$\mathrm{CD}^{+} \mathrm{T}$ helper (Th) cells play a central role in orchestrating immune responses to diverse microbial pathogens [1]. Upon activation by antigens, naive $\mathrm{CD} 4^{+} \mathrm{T}$ cells differentiate into specialized effector T (Teff) cells (Th1, Th2, or Th17), which secrete different patterns of cytokines and perform different functions [1]. Th1 cells produce interferon- $\gamma($ IFN- $\gamma)$ and tumor necrosis factor- $\alpha$ (TNF- $\alpha)$ and initiate cellular immune responses against intracellular pathogens. Th2 cells generate interleukin-4 (IL-4), IL-5 and IL-13 and promote humoral responses against extracellular parasites. Th17 cells make IL-17, IL-21 and IL-22 and confer immunity against extracellular bacteria and fungi. Moreover, activated $\mathrm{CD} 4^{+} \mathrm{T}$ cells also differentiate into regulatory $\mathrm{T}$ (Treg) cells, which express transforming growth factor- $\beta$ (TGF- $\beta$ ), IL-10 and IL-35 and suppress the functions of Teff cells, thereby keeping immune responses in check.

Imbalance of Th cell differentiation and subsequent cytokine dysregulation is implicated in inflammatory and

\footnotetext{
* Correspondence: xiaog2@upmc.edu

${ }^{1}$ University of Pittsburgh Cancer Institute, Pittsburgh, PA, USA

Department of Microbiology and Molecular Genetics, Pittsburgh, PA, USA

Full list of author information is available at the end of the article
}

autoimmune diseases [2]. In particular, Th1 and Th17 cells and their signature cytokines IFN- $\gamma$ and IL-17 have been shown to play a critical role in the development of autoimmune responses in many autoimmune diseases, including multiple sclerosis (MS) and rheumatoid arthritis [2-4]. In accordance with the significance of Th cell differentiation in animal physiology and pathology, the molecular mechanisms underlying this important process have been extensively investigated. In this regard, the signal transducers and activators of transcription (STAT) proteins are well known for their essential roles in transmitting cytokine-mediated signals and specifying Th cell differentiation $[1,2]$. In general, STAT4 is activated mainly by IL-12 and type I IFNs, and it functions predominantly in promoting Th1 cell differentiation. STAT6 is activated in response to IL-4 and functions as the molecular switch for initiation of the Th2 cell differentiation program. Soon after activation by IL-6, STAT3 triggers Th17 commitment. On the other hand, IL2-activated STAT5 facilitates Treg cell differentiation. Similar to STAT proteins, the NF-kB transcription factors, particularly the prototypical member RelA (also known as p65), are also master regulators/activators of immune responses and inflammation in both healthy and disease $[5,6]$. The signaling pathways leading to activation of STAT

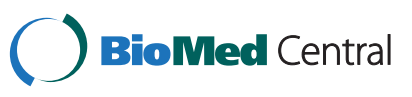


and NF- $\mathrm{kB}$ proteins have been well demonstrated $[7,8]$. However, it still remains largely unknown how activated STAT and NF- $\mathrm{B}$ are terminated for proper Th cell differentiation and immune responses and how STAT and NF$\mathrm{\kappa B}$ are deregulated in autoimmune diseases.

Previous studies show that PDLIM2, a ubiquitously expressed PDZ-LIM domain-containing protein with high expression in lymphoid tissues and cells including $\mathrm{T}$ lymphocytes, is required for the termination of STAT and NF- $\mathrm{kB}$ activation $[9,10]$. More recent studies suggest that PDLIM2 may function as a tumor suppressor [11-15]. Mechanistic studies indicate that PDLIM2 selectively promotes ubiquitination and proteasomal degradation of nuclear (activated) STAT4 and RelA proteins [9-12]. However, whether and how PDLIM2 is involved in Th cell differentiation remain unknown. In particular, mouse genetic studies reveal that PDLIM2 is not required for the development of immune cells and immune tissues/organs [9]. Additionally, it remains unknown whether PDLIM2 is involved in the pathogenesis of inflammatory and autoimmune diseases.

\section{Results and discussion}

PDLIM2 deficiency in CD4 ${ }^{+}$th cells enhances Th1 and Th17 cell differentiation but has no obvious effect on Th2 and Treg cell differentiation

To test whether PDLIM2 is involved in Th cell differentiation, naive $\mathrm{CD}^{+}$Th cells were isolated from spleens of
PDLIM2 $^{-/-}$and PDLIM2 ${ }^{+/+}$mice and stimulated by antiCD3/anti-CD28 under Th1, Th2, Th17 or Treg polarizing condition. Loss of PDLIM2 did not affect the differentiation of Th cells to Th2 or Treg, as evidenced by similar numbers of Th2 and Treg cells produced from naive PDLIM2 $2^{-/}$and PDLIM2 $^{+/+} \mathrm{CD}^{+}$Th cells (Figure 1 ). In contrast, much more Th1 and Th17 cells were generated from naive PDLIM2 ${ }^{-/-} \mathrm{CD}^{+}$Th cells compared to PDLIM2 ${ }^{+/+}$cells. These data suggest that PDLIM2 plays a specific role in restricting Th1 and Th17 cell differentiation.

\section{Mice deficient in PDLIM2 show increased susceptibility to EAE}

Given the causative role of Th1 and Th17 cells in autoimmune diseases such as MS [2-4], we proposed that through restriction of Th1 and Th17 cell differentiation, PDLIM2 is involved in autoimmune disease suppression. To test this hypothesis and to further characterize the in vivo role of PDLIM2 in regulating Th1 and Th17 cell differentiation, we examined the susceptibility of $\mathrm{PDLIM}^{-/-}$and PDLIM2 ${ }^{+/+}$mice to EAE, a well-defined model of MS [16]. In agreement with previous studies [17], 20\% of PDLIM2 $2^{+/+}$mice developed acute EAE with a 2.8 mean peak clinical score and a mean disease onset of day $17.3 \pm 2.5$ ) of post-immunization with the encephalitogenic $\mathrm{PLP}_{180-199}$ epitope (Figure 2). Remarkably, over 50\% of PDLIM2 $2^{-/-}$mice developed EAE with an earlier disease onset $(13.1 \pm 1.9$ day of post-immunization) and a more

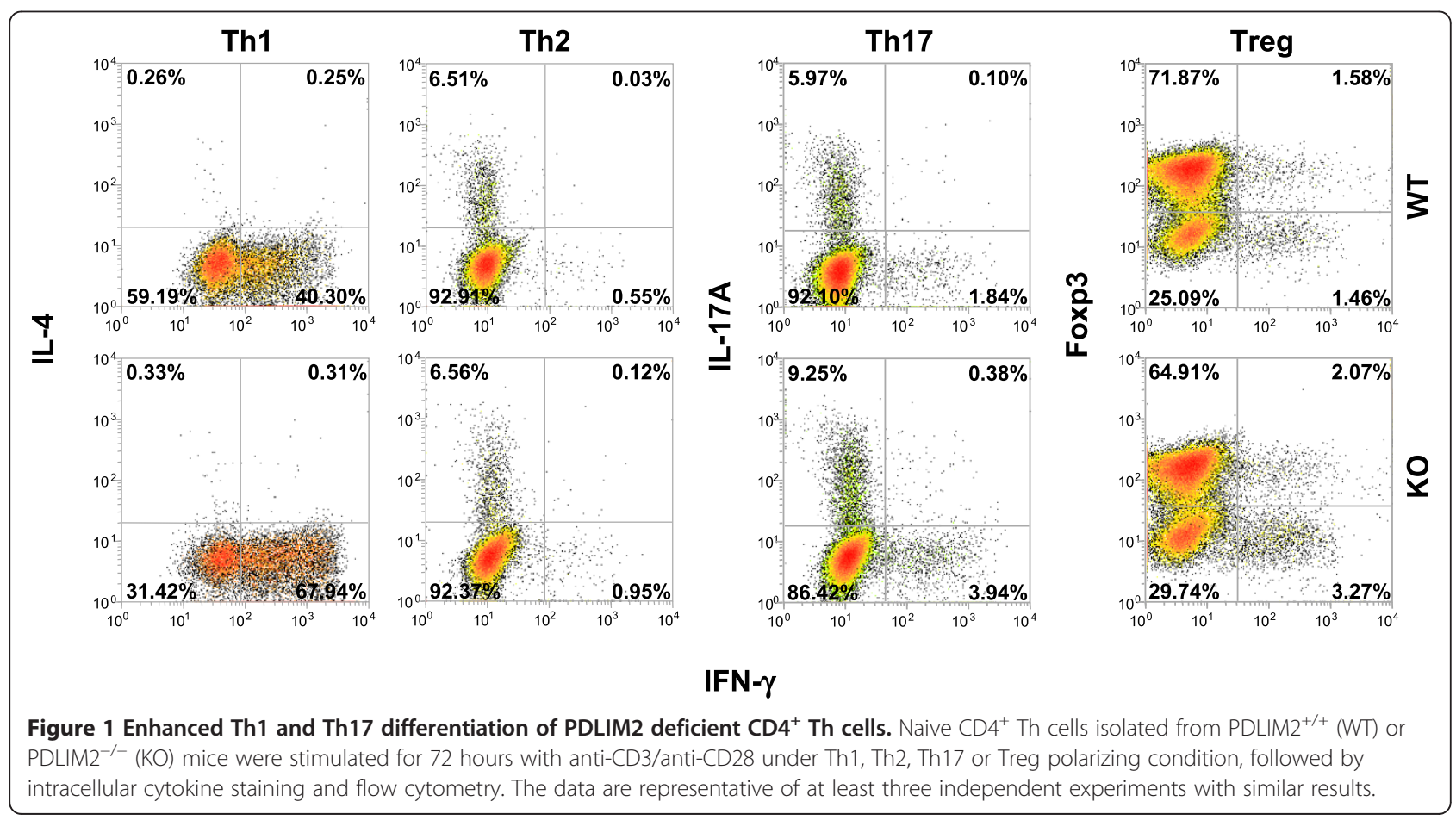



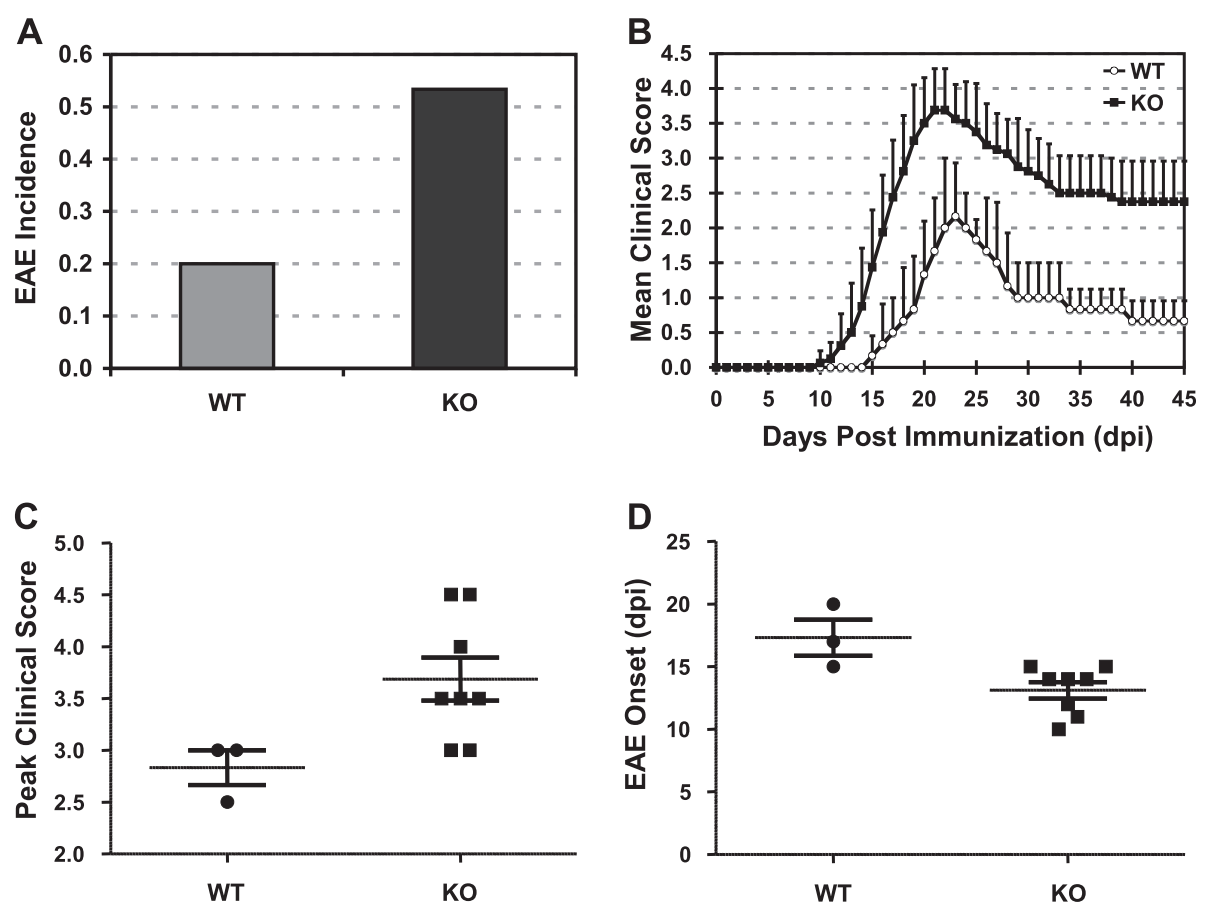

Figure $\mathbf{2}$ Increased susceptibility to EAE in PDLIM2 deficient mice. A) Incidence, B) disease progression, C) severity and D) onset of EAE in $\mathrm{PDLIM2}^{+/+}$and PDLIM2 ${ }^{-/-}$mice $(n=15)$. Mice were immunized with PLP $180-199$ peptide and monitored daily for EAE disease symptoms. The $p$ values between the PDLIM2 ${ }^{+/+}(\mathrm{WT})$ and $\mathrm{PDLIM}^{-/-}(\mathrm{KO})$ groups are at least smaller than 0.05 by two tailed t-test.

severe (3.7 mean peak clinical score) and prolonged disease course. These data clearly indicate that PDLIM2 plays a critical role in suppressing EAE.

\section{PDLIM2 expression in $\mathrm{CD}^{+}{ }^{+} \mathrm{T}$ cells is critical for EAE suppression}

To determine whether the effect of PDLIM2 deficiency on EAE is $\mathrm{CD}^{+}$T-cell specific, we performed adoptive $\mathrm{CD}^{+}$T-cell transfer studies using SCID mice as receipts, which lack $\mathrm{CD}^{+}{ }^{+} \mathrm{T}$ cells. Although the disease severity in adoptive transfer recipients was less robust overall than that observed in immunized mice, the difference of EAE induction in the receipts of PDLIM2 $2^{+/+}$ versus PDLIM2 ${ }^{-1-} \mathrm{T}$ cells was still significant and similar to that observed in PDLIM2 $2^{+/+}$and PDLIM2 $2^{-/-}$mice (Figure 3). These data suggest that the observed increase in EAE severity in PDLIM2 $2^{-/-}$mice is due to the deficiency of PDLIM2 in CD4 ${ }^{+} \mathrm{T}$ cells.

\section{PDLIM2 deficiency leads to increased STAT and NF-KB activation and augmented production of Th1 and Th17 cytokines}

As EAE is mediated by Th1 and/or Th17 cells [3], we examined whether the exacerbated EAE in PDLIM2 ${ }^{-/-}$ mice is associated with increased Th1 and Th17 cell differentiation in the mice. As expected, the expression levels of Th1 cytokines (IFN- $\gamma$ and TNF- $\alpha$ ) and Th17 cytokines (IL-17, IL-21 and IL-22) were significantly higher in $\mathrm{PLP}_{180-199^{-}}$stimulated $\mathrm{PDLIM}^{-/-}$mice compared to the PDLIM2 $2^{+/+}$mice under the same treatment (Figure 4A). On the other hand, the expression levels of Th2 cytokines (IL-4, IL-5 and IL-13) and Treg cytokines (TGF- $\beta$ and IL-10) were comparable in the PLP $180-199^{-}$ treated $\mathrm{PDLIM}^{+/+}$or $\mathrm{PDLIM} 2^{-/-}$mice. These data

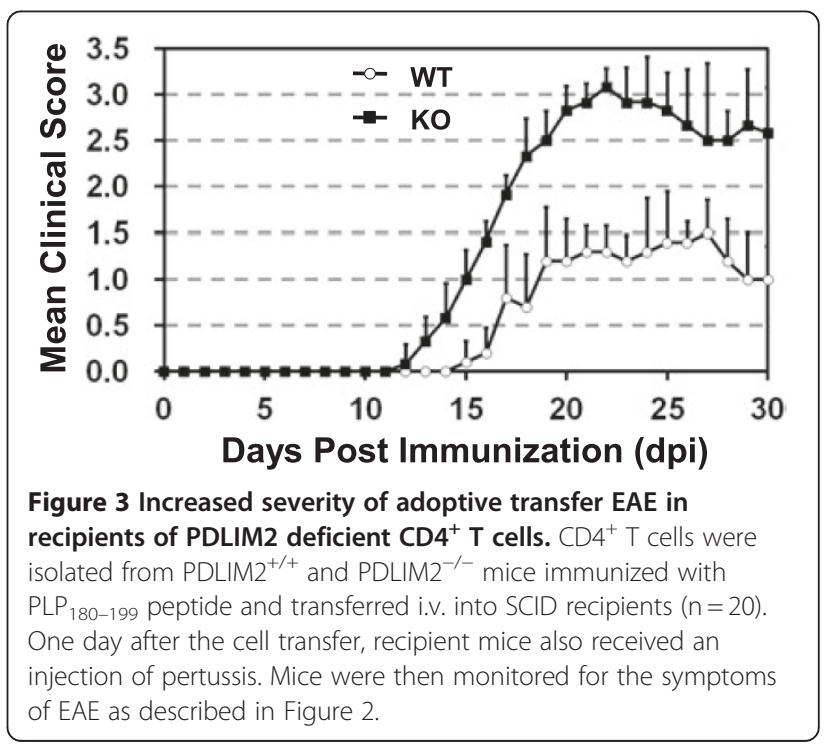



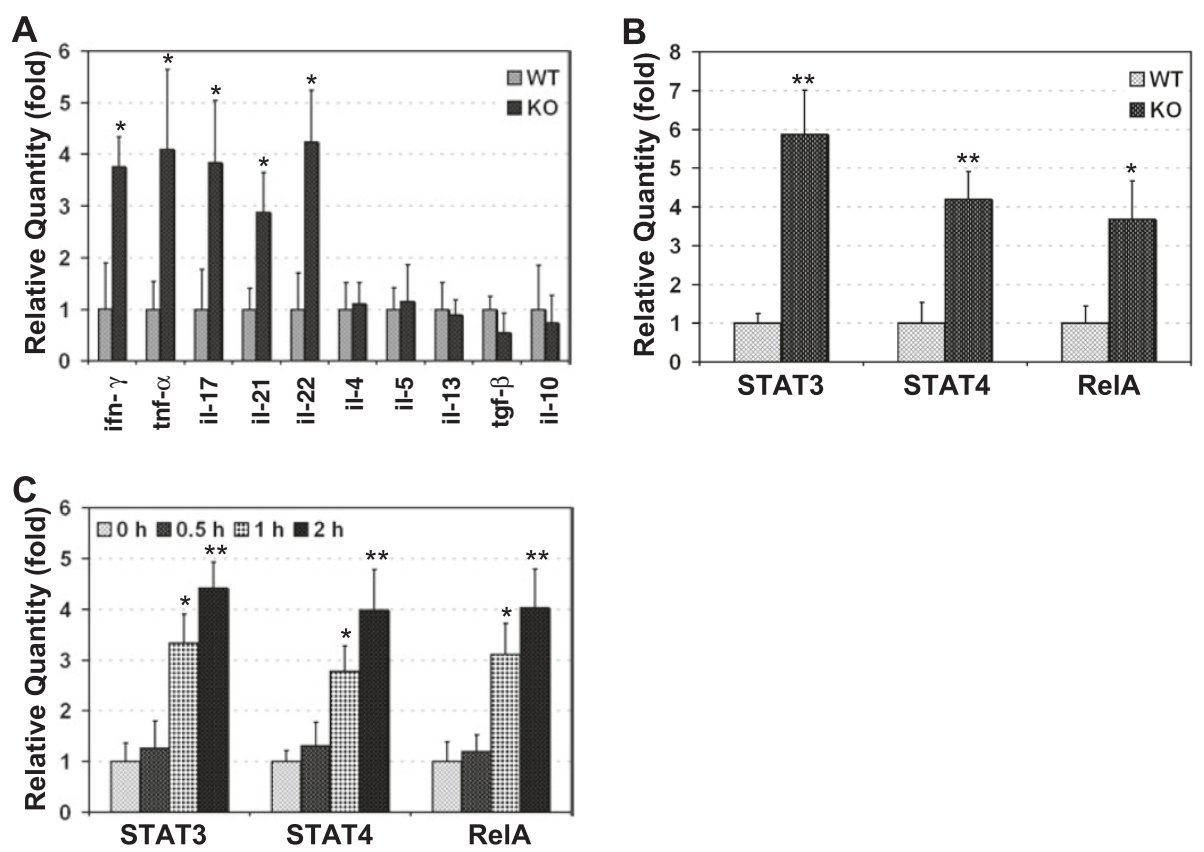

Figure 4 Enhanced nuclear expression of STAT3/4 and RelA proteins and augmented production of Th1 and Th17 cytokines in PDLIM2 deficient Teff cells. Splenic T cells from day 10 PLP $180-199$-immunized PDLIM2 ${ }^{+/+}$(WT) or PDLIM2 ${ }^{-1-}(\mathrm{KO})$ mice were subjected to QRT-PCR to detect the relative expression levels of the indicated cytokines genes (A) or ELISA to detect the nuclear expression levels of STAT3, STAT4 and RelA (B). The expression levels of the indicated genes and proteins were represented as fold induction relative to their WT controls. C) Naive $\mathrm{PDLIM2}^{-/-}$or PDLIM2 ${ }^{+/+} \mathrm{CD}^{+}$Th cells were stimulated for the indicated time points with anti-CD3/anti-CD28 under Th1 or Th17 polarizing condition, followed by ELISA to detect the nuclear expression levels of STAT3 (in response to Th17 stimulation), STAT4 and RelA (in response to Th1 stimulation). In A-C, $n=3,{ }^{*}, p<0.03 ;{ }^{* *}, p<0.003$ by two tailed $t$-test.

suggest that PDLIM2 suppresses EAE through limiting Th1 and Th17 cell differentiation.

To determine the molecular mechanisms by which PDLIM2 controls Th1 and Th17 cell differentiation for EAE suppression, we examined the expression levels of STAT4 and RelA proteins in the nucleus (activation marker) of CD4 ${ }^{+} \mathrm{T}$ cells isolated from $\mathrm{PLP}_{180-199}$-treated $\mathrm{PDLIM}^{+/+}$mice or PDLIM2 ${ }^{-/-}$mice. In this regard, it is known that PDLIM2 promotes proteasomal degradation of nuclear STAT4 and RelA proteins [9-12]. More importantly, STAT4 is a determinative factor of Th1 cell differentiation and also participates in Th17 cell differentiation $[18,19]$. On the other hand, RelA regulates transcriptional expression of numerous cytokines that are involved in Th1 and Th17 cell differentiation and EAE pathogenesis such as IFNs, TNF- $\alpha$ and IL-6 [6]. In fact, a recent study has already linked RelA to Th17 response [20]. Given the critical role of STAT3 in Th17 cell differentiation [21], we also included STAT3 in our studies. As shown in Figure 4B, significantly higher levels of STAT3, STAT4 and RelA proteins were detected in $\mathrm{PLP}_{180-199^{-}}$treated T cells from PDLIM2 ${ }^{-/-}$mice as compared to those from PDLIM2 ${ }^{+/+}$mice. The increased nuclear expression/activation of STAT3, STAT4 and RelA should be the driving force but not the consequences of enhanced Th1 and Th17 cell differentiation nor the outcome of exacerbated EAE in PDLIM2 ${ }^{-1-}$ mice, because an obvious increase in the nuclear expression of STAT3, STAT4 and RelA proteins was already detected within 30 minutes after cell stimulation (Figure 4C). Our biochemical studies indicated that similar to its role in the negative regulation of STAT4 and RelA (9-12), PDLIM2 bound to nuclear STAT3 for ubiquitination and proteasomal degradation (Figure 5). During the preparation of our manuscript, another group also showed that PDLIM2 targets STAT3 for degradation [22]. These data together suggest that PDLIM2 negatively regulates activation of STAT3/4 and RelA and therefore restricts Th1 and Th17 cell differentiation and prevents EAE development.

The STAT and NF- $\mathrm{B}$ transcription factors play critical roles at multiple levels of the immune system in both health and disease, including the autoimmune inflammatory response [1-6]. The mechanisms of how STAT and $\mathrm{NF}-\mathrm{kB}$ are activated to drive immune responses have been well defined $[7,8]$. However, how those key immune regulators are negatively regulated during Th cell differentiation and how they become constitutively and persistently activated in autoimmune diseases remain largely unknown. The data presented in this study demonstrate that PDLIM2 functions as an essential 

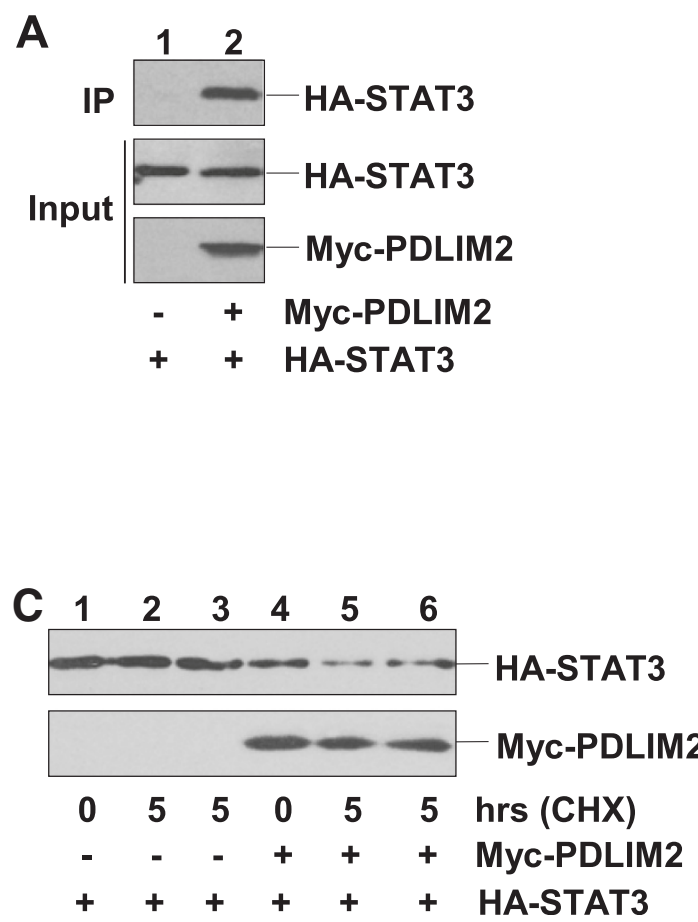

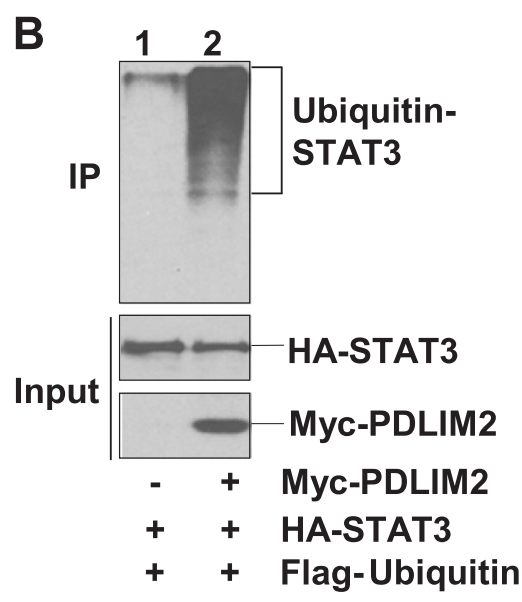

Figure 5 Ubiquitination and proteasomal degradation of STAT3 by PDLIM2. A) Physical interaction between PDLIM2 and STAT3. Nuclear extracts of 293 cells transfected with HA-STAT3 alone or together with Myc-PDLIM2 were subjected to immunoprecipitation (IP) using Myc antibody and immunoblotting (IB) using HA antibody. The expression levels of HA-STAT3 and Myc-PDLIM2 were examined by IB. B) Polyubiquitination of STAT3 by PDLIM2. 293 cells were transfected with HA-STAT3 plus Flag-ubiquitin in the presence or absence of Myc-PDLIM2, followed by nuclear fractionation. The nuclear extracts were subjected to IP using HA antibody and IB using Flag antibody. The expression levels of HA-STAT3 and Myc-PDLIM2 were examined by IB. C) Proteasomal degradation of STAT3 by PDLIM2. 293 cells transfected with HA-STAT3 alone or together with Myc-PDLIM2 were cycloheximide (CHX)-chased for the indicated time, followed by nuclear extractions and IB using HA or Myc antibody. In lanes 3 and 6, the cells were chased in the presence of $10 \mu \mathrm{M}$ MG132.

modulator of Th1 and Th17 cell differentiation but has no apparent effect on Th2 and Treg cell differentiation. Interestingly, the novel function of PDLIM2 in Th cell differentiation is most likely through restricting activation of STAT3/4 and RelA. These data identify STAT3 as a new target of PDLIM2 for ubiquitin-mediated proteasomal degradation and also suggest a new mechanism of RelA in immune responses involving regulation of Th1 and Th17 cell differentiation. These findings provide important insights into molecular mechanisms underlying immune responses and suggest PDLIM2 as a new therapeutic target for inflammatory and autoimmune diseases.

\section{Methods}

Mice

$\mathrm{PDLIM}^{-/-}$mice were backcrossed with $\mathrm{BALB} / \mathrm{c}$ mice at least 10 generations for pure $\mathrm{BALB} / \mathrm{c}$ background. $\mathrm{PDLIM}^{-/-}$BALB/C mice and control PDLIM $2^{+/+}$ $\mathrm{BALB} / \mathrm{c}$ mice were housed under specific pathogen-free conditions at the Hillman Cancer Center of the University of Pittsburgh Cancer Institute. Animal experiments were approved by the Institutional Animal Care and Use Committee (IACUC) of the University of Pittsburgh.

\section{Experimental autoimmune encephalitis (EAE) induction} and clinical scoring

Six to eight-week-old female mice were immunized subcutaneously with $\mathrm{PLP}_{180-199}$ peptide $(200 \mu \mathrm{g} / \mathrm{mouse}$, Genemed Synthesis Inc.) emulsified in CFA containing Mycobacterium tuberculosis H37Ra (500 $\mu \mathrm{g} /$ mouse, BD Diagnostics). Mice also received $300 \mathrm{ng}$ of pertussis toxin (List Biological Laboratories) intraperitoneally (i.p.) at the time of immunization and 48 hours later. Mice were monitored daily for clinical signs of paralysis and scored as follows: 0 , no clinical signs; 1 , limp tail; 2 , weak/partially paralyzed hind legs; 3, limp tail and complete paralysis of hind legs; 4, complete hind and partial front leg paralysis; 5 , complete paralysis or moribund state.

\section{Adoptive transfer of $\mathrm{CD}^{+}{ }^{+} \mathrm{T}$ cells for induction of EAE}

Lymph nodes and spleens were harvested from PDLIM2 ${ }^{+/+}$ or PDLIM2 $^{-/-}$mice immunized with PLP $180-199$, and lymph node cells and splenocytes were cultured in vitro 
with $1 \mu \mathrm{M}$ PLP ${ }_{180-199}$ and IL-2 for 72 h. CD $4^{+}$T cells were then positively selected by MACS separation using magnetic $\mathrm{CD} 4^{+}$microbeads (Miltenyi Biotec, Auburn, CA) per manufacturer's instructions. $5 \times 10^{6} \mathrm{CD} 4^{+} \mathrm{T}$ cells were adoptively transferred by intravenous (i.v.) injection into SCID recipients on day 0 . On day 2, mice received an i.p. injection of pertussis toxin (250 ng), and mice were then monitored for symptoms of disease.

\section{$\mathrm{CD}^{+}$th cell purification and in vitro differentiation}

Naive $\mathrm{CD}^{+} \mathrm{CD} 25^{-} \mathrm{T}$ cells were first isolated from splenocytes using $\mathrm{CD}^{+}{ }^{+} \mathrm{T}$-cell Isolation Kit (Miltenyi Biotec.) and then sorted out by FACSAria (BD Biosciences). Purified naive $\mathrm{CD} 4^{+} \mathrm{CD} 25^{-} \mathrm{T}$ cells were stimulated with plate-bound anti-CD3 and anti-CD28 $(1 \mu \mathrm{g} / \mathrm{ml})$ under Th1 (mIL-2 $10 \mathrm{ng} / \mathrm{ml}, \mathrm{mIL}-1210 \mathrm{ng} / \mathrm{ml}$ ), Th2 (IL-4 $10 \mathrm{ng} / \mathrm{ml}$, antiIFN $\gamma 10 \mu \mathrm{g} / \mathrm{ml}$ ), Th17 (anti-IFN $\gamma 10 \mu \mathrm{g} / \mathrm{ml}$, anti-IL-4 $10 \mu \mathrm{g} / \mathrm{ml}$, hIL-6 $10 \mathrm{ng} / \mathrm{ml}$, hTGF- $\beta 10 \mathrm{ng} / \mathrm{ml}$ ) or Treg (hTGF $\beta, 10 \mathrm{ng} / \mathrm{ml}$, anti-IL-4 $10 \mu \mathrm{g} / \mathrm{ml}$, anti-IFN $10 \mu \mathrm{g} / \mathrm{ml}$ ) (BD Biosciences or eBioscience) polarizing condition. 72 hours after the initial stimulation, the cells were subjected to intracellular cytokine staining (ICS)/flow cytometry analysis and quantitative real-time RT-PCR (QRT-PCR) as described below.

\section{ICS and flow cytometry}

$\mathrm{T}$ cells were stimulated for 5 hours with PMA $(50 \mathrm{ng} / \mathrm{ml})$ and ionomycin $(500 \mathrm{ng} / \mathrm{ml})$ in the presence of intracellular transport inhibitor monesin $(10 \mu \mathrm{g} / \mathrm{ml}$; Sigma), followed by fixation with paraformaldehyde (2\%) and permeablization with saponin $(0.5 \%)$. Cells were then treated with anti-IFN- $\gamma$-FITC (XMG1.2), anti-IL-4-PE (11B11), antiIL-17-PE (TC11-18 H10), and anti-Foxp3-FITC (FJK16 s) (BD Biosciences or eBioscience). Data were acquired using FACSCalibur (BD Biosciences) and analyzed using CellQuest software (Becton Dickinson) as described previously [23].

\section{QRT-PCR}

Total RNA was prepared with TRIZOL reagent and cDNA was generated with SuperScript II reverse transcriptase (Invitrogen), followed by real-time PCR assays using Fast start SYBR Green reagents (Roche) as described $[24,25]$. The gene-specific primer pairs were: IFN- $\gamma$, 5'-TTCTTCAGCAACAGCAAGGCGAA-3' and 5'-TGAATGCTTGGCGCTGGACCTG-3'; TNF- $\alpha$, 5'-G ATGAGAAGTTCCCAAATGGC-3' and 5'-ACTTGGT GGTTTGCTACGACG-3'; TGF- $\beta$, 5'-TGACGTCACT GGAGTTGTACGG-3' and 5'-GGTTCATGTCATGGAT GGTGC-3'; IL-4, 5'-AGGGACGCCATGCACGGAGAT3' and 5'-GCGAAGCACCTTGGAAGCCCTAC-3'; IL-5, 5'-AGCACAGTGGTGAAAGAGACCTT-3' and 5'-TCC AATGCATAGCTGGTGATTT-3'; IL-10, 5'-AGCTGAA GACCCTCAGGATGCG-3' and 5'- TCATTCATGGCC
TTGTAGACACCTTG-3'; IL-13, 5'-GGCTCTTGCTTG CCTTGGTG-3' and 5'-TCCATACCATGCTGCCGTT G-3'; IL-17, 5'-CTCAGACTACCTCAACCGTTC-3' and 5'-TGAGCTTCCCAGATCACAGAG-3'; IL-21, 5'-ATCC TGAACTTCTATCAGCTCCAC-3' and 5'-GCATTTAG CTATGTGCTTCTGTTTC-3'; IL-22, 5'-TCCGAGGAG TCAGTGCTAAA-3' and 5'AGAACGTCTTCCAGGG TGAA-3'; $\beta$-actin, 5 '-ACCCGCGAGCACAGCTTCTT TG-3' and 5'-CTTTGCACATGCCGGAGCCGTTG-3'. Expression levels of each gene were normalized to that of $\beta$-actin.

\section{Enzyme-linked immunosorbent assay (ELISA)}

Cell nuclear fractions were prepared and added to 96-well plate precoated with anti-RelA, anti-STAT3 or antiSTAT4. After overnight incubation at $4{ }^{\circ} \mathrm{C}$, plates were washed extensively with PBS containing $0.1 \%$ Tween 20 (PBST), and horseradish peroxidase-conjugated secondary antibodies were added and incubated for 1 hour at room temperature. After extensive wash with PBST, a colorimetric substrate 2'2-azinobis(3-ethylenzthiazoline-6-sulfonic acid) (ABTS) was added and incubated for 15 minutes. The reaction was stopped by addition of $100 \mu \mathrm{L} 1 \%$ sodium dodecyl sulfate (SDS). The optical density at $405 \mathrm{~nm}$ (OD405) was measured with an automated plate spectrophotometer (Thermo Lab Systems).

\section{Immunoblotting (IB) and immunoprecipitation (IP) assays} Nuclear extracts were subjected to SDS-PAGE and IB, or IP using the indicated antibodies before SDS-PAGE and IB as described before [26,27].

\section{In vivo ubiquitin conjugation assay}

Cytoplasmic and nuclear extracts were prepared from HTLV-I-transformed T cells or 293 cells transfected with HA-STAT3 together with Flag-tagged ubiquitin in the presence or absence of Myc-PDLIM2, immediately followed by IP using anti-HA. The ubiquitin-conjugated STAT3 pulled down by IP was detected by IB using antiFlag [28].

\section{Protein stability assay}

Cells were treated with $10 \mu \mathrm{M} \mathrm{CHX}$, followed by chase of the indicated time period in the presence or absence of MG132, and IB to detect the indicated proteins [29].

\section{Statistical analysis}

Data were reported as mean \pm standard deviation (SD). The Student's $t$ test (two tailed) was used to assess significance of differences between two groups, and $\mathrm{p}$ values $\leq 0.05$ and 0.01 were considered statistically significant and highly statistically significant, respectively. 


\section{Abbreviations}

ABTS: 2'2-azinobis(3-ethylenzthiazoline-6-sulfonic acid); EAE: Experimental autoimmune encephalitis; ELISA: Enzyme-linked immunosorbent assay; IB: Immunoblotting (IB); ICS: Intracellular cytokine staining; IFN- $\gamma$ : Interferon- $\gamma$; IL: Interleukin; IP: Immunoprecipitation; (i.p.): Intraperitoneal; (i.v.): Intravenous; MS: Multiple sclerosis; QRT-PCR: Quantitative reverse transcriptionpolymerase chain reaction; PDLIM2: PDZ-LIM domain-containing protein 2; STAT: Signal transducers and activators of transcription; SDS: Sodium dodecyl sulfate; TGF- $\beta$ : Transforming growth factor- $\beta$; Th: T helper; Teff: Effector T; TNF-a: Tumor necrosis factor- $a$.

\section{Competing interest}

The authors declare that they have no competing interests.

\section{Authors' contributions}

$\mathrm{ZQ}, J \mathrm{~F}, \mathrm{HM}, \mathrm{JZ}$ and MJ performed experiments; MM analyzed data and criticized the paper; MG contributed vital new reagents and criticized the paper; ZQ and GX designed the research, analyzed data and wrote the paper. All authors read and approved the final manuscript.

\section{Acknowledgments}

The authors thank J.A. Lyons for critical technical assistance. This study was supported in part by the National Institute of Health (NIH)/National Cancer Institute (NCI) grant R01 CA116616 and American Cancer Society (ACS) Awards RSG-06-066-01-MGO (G. X.) and PF-12-081-01-TBG (Z. Q.). M.Y.M and H.M were supported in part by NHLBI grant RO1 HL093716 and RO1GM063569. This project also used the UPCI shared co-facilities supported in part by the $\mathrm{NIH} / \mathrm{NCl}$ grant P30CA047904

\section{Author details}

${ }^{1}$ University of Pittsburgh Cancer Institute, Pittsburgh, PA, USA. ${ }^{2}$ Department of Microbiology and Molecular Genetics, Pittsburgh, PA, USA. ${ }^{3}$ Department of Medicine, University of Pittsburgh School of Medicine, Pittsburgh, PA, USA. ${ }^{4}$ Department of Immunology and Infectious Diseases, Harvard School of Public Health, Boston, MA, USA.

Received: 21 March 2012 Accepted: 6 June 2012

Published: 25 June 2012

\section{References}

1. O'Shea JJ, Paul WE: Mechanisms underlying lineage commitment and plasticity of helper CD4+ T cells. Science 2010, 327:1098-1102.

2. Jäger $A$, Kuchroo VK: Effector and regulatory T-cell subsets in autoimmunity and tissue inflammation. Scand J Immunol 2010, 72:173-184

3. Fletcher JM, Lalor SJ, Sweeney CM, Tubridy N, Mills KH: T cells in multiple sclerosis and experimental autoimmune encephalomyelitis. Clin Exp Immunol 2010, 162:1-11.

4. Toh ML, Miossec P: The role of T cells in rheumatoid arthritis: new subsets and new targets. Curr Opin Rheumatol 2007, 19:284-288.

5. Xiao G, Rabson A, Young W, Qing G, Qu Z: Alternative pathways of NF-kB activation: a double-edged sword in health and disease. Cytokine Growth Factor Rev 2006, 17:281-293.

6. Brown KD, Claudio E, Siebenlist U: The roles of the classical and alternative nuclear factor-KB pathways: potential implications for autoimmunity and rheumatoid arthritis. Arthritis Res Ther 2008, 10:212.

7. Santos Cl, Costa-Pereira AP: Signal transducers and activators of transcription-from cytokine signaling to cancer biology. Biochim Biophys Acta 2011, 1816:38-49.

8. Xiao G, Fu J: NF-KB and cancer: a paradigm of Yin-Yang. Am J Cancer Res 2011, 1:192-221

9. Tanaka T, Soriano MA, Grusby MJ: SLIM is a nuclear ubiquitin E3 ligase that negatively regulates STAT signaling. Immunity 2005, 22:729-736.

10. Tanaka T, Grusby MJ, Kaisho T: PDLIM2-mediated termination of transcription factor NF-KB activation by intranuclear sequestration and degradation of the p65 subunit. Nat Immunol 2007, 8:584-591.

11. Qu Z, Fu J, Yan P, Hu J, Cheng S, Xiao G: Epigenetic repression of PDLIM2: implications for the biology and treatment of breast cancer. J Biol Chem 2010, 285:11786-11792.

12. Qu Z, Yan P, Fu J, Jiang J, Grusby MJ, Smithgall TE, Xiao G: DNA methylation-dependent repression of PDLIM2 in colon cancer and its role as a potential therapeutic target. Cancer Res 2010, 70:1766-1772.
13. Yan P, Fu J, Qu Z, Li S, Tanaka T, Grusby MJ, Xiao G: PDLIM2 suppresses HTLV-I Tax-mediated tumorigenesis by targeting Tax into the nuclear matrix for proteasomal degradation. Blood 2009, 113:4370-4380

14. Yan P, Qu Z, Li S, Ishikawa C, Mori N, Xiao G: HTLV-I-mediated repression of PDLIM2 involves DNA methylation but independent of the viral oncoprotein Tax. Neoplasia 2009, 11:1036-1041.

15. Fu J, Yan P, Li S, Qu Z, Xiao G: Molecular determinants of PDLIM2 in suppressing HTLV-I Tax-mediated tumorigenesis. Onocogene 2010, 29:6499-4507.

16. Morel L: Mouse models of human autoimmune diseases: essential tools that require the proper controls. PLoS Biol 2004, 2:E241.

17. Lyons JA, Ramsbottom MJ, Mikesell RJ, Cross AH: B cells limit epitope spreading and reduce severity of EAE induced with PLP peptide in BALB/c mice. J Autoimmun 2008, 31:149-155.

18. Nishikomori R, Usui T, Wu CY, Morinobu A, O'Shea JJ, Strober W: Activated STAT4 has an essential role in Th1 differentiation and proliferation that is independent of its role in the maintenance of IL-12R beta 2 chain expression and signaling. J Immunol 2002, 169:4388-4398.

19. Mathur AN, Chang HC, Zisoulis DG, Stritesky GL, Yu Q, O'Malley JT, Kapur R, Levy DE, Kansas GS, Kaplan MH: Stat3 and Stat4 direct development of IL-17-secreting Th cells. J Immunol 2007, 178:4901-4907.

20. Ruan Q, Kameswaran V, Zhang Y, Zheng S, Sun J, Wang J, DeVirgiliis J, Liou HC, Beg AA, Chen YH: The Th17 immune response is controlled by the RelRORY-RORY T transcriptional axis. J Exp Med 2011, 208:2321-2333.

21. Harris TJ, Grosso JF, Yen HR, Xin H, Kortylewski M, Albesiano E, Hipkiss EL, Getnet D, Goldberg MV, Maris CH, Housseau F, Yu H, Pardoll DM, Drake CG: Cutting edge: an in vivo requirement for STAT3 signaling in TH17 development and TH17-dependent autoimmunity. J Immunol 2007, 179:4313-4317.

22. Tanaka T, Yamamoto Y, Muromoto R, lkeda O, Sekine Y, Grusby MJ, Kaisho T, Matsuda T: PDLIM2 inhibits T helper 17 cell development and granulomatous inflammation through degradation of STAT3. Sci Signal 2011, 4:ra85.

23. Qu Z, Sun D, Young W: Lithium promotes neural precursor cell proliferation: evidence for the involvement of the non-canonical GSK-3ßNF-AT signaling. Cell Biosci 2011, 1:18.

24. Fu J, Qu Z, Yan P, Ishikawa C, Ageilan RI, Rabson AB, Xiao G: The tumor suppressor gene WWOX links the canonical and noncanonical NF-KB pathways in HTLV-I Tax-mediated tumorigenesis. Blood 2011, 117:1652-1661.

25. Qing G, Qu Z, Xiao G: Endoproteolytic processing of C-terminally truncated NF-KB2 precursors at KB-containing promoters. Proc Natl Acad Sci U S A 2007, 104:5324-5329.

26. Qing G, Qu Z, Xiao G: Regulation of NF-KB2 $\mathrm{p} 100$ processing by its cisactivating domain. J Biol Chem 2005, 280:18-27.

27. Qing G, Yan P, Xiao G: Hsp90 inhibition results in autophagy-mediated proteasome-independent degradation of IKB kinase (IKK). Cell Res 2006, 16:895-901.

28. Qu Z, Qing G, Rabson R, Xiao G: Tax deregulation of NF-KB2 p100 processing involves both $\beta$ - $T r C P$-dependent and independent mechanisms. J Biol Chem 2004, 279:44563-44572.

29. Qing G, Qu Z, Xiao G: Stabilization of basally translated NF-kB-inducing kinase (NIK) protein functions as a molecular switch of processing of NF-kB2 p100. J Biol Chem 2005, 280:40578-40582.

doi:10.1186/2045-3701-2-23

Cite this article as: Qu et al: PDLIM2 restricts Th1 and Th17

differentiation and prevents autoimmune disease. Cell \& Bioscience 2012 2:23. 